\title{
Review
}

\section{Combination Therapy with Aldosterone Blockade and Renin-Angiotensin Inhibitors Confers Organ Protection}

\author{
Atsuhisa SATO ${ }^{1)}$, Takao SARUTA ${ }^{2)}$, and John W. FUNDER ${ }^{3)}$
}

\begin{abstract}
There is increasing evidence that aldosterone exerts major adverse cardiovascular effects through classical mineralocorticoid receptors (MR) in nonepithelial tissues such as the brain and heart. This nonepithelial role of aldosterone has been underscored by the recent Randomized Aldactone Evaluation Study (RALES) and the Eplerenone Post-AMI Heart Failure Efficacy and Survival Study (EPHESUS). These studies also showed that when using MR antagonist as an "organ protecting" drug, further organ protection could be derived by the addition of an angiotensin-converting enzyme (ACE) inhibitor or angiotensin II type 1 receptor blocker (ARB). The long-term effect of aldosterone was not inhibited in some subjects, so the possibility of organ damage due to so-called "breakthrough" aldosterone cannot be ignored. Nonepithelial MR-mediated effects played a major role in this aldosterone effect. These effects can be inhibited by MR antagonist at a small dose, not lower blood pressure. Therefore, the idea is now to combine a small dose of MR antagonist with an ACE inhibitor or ARB. However, warnings have been given recently due to the emergence of hyperkalemia and other adverse effects associated with inappropriate combination therapy. It is important to note that, if the eligibility criteria of RALES and EPHESUS are fulfilled, the potassium level will rarely become problematic. Therefore, the recent increase in the incidence of adverse effects can be attributed to the incorrect application of combination therapy. Elderly patients or those with dehydration, renal dysfunction, and aggravated heart failure require further close monitoring or termination of spironolactone administration. The combination of an MR antagonist and renin-angiotensin inhibitors should be a useful strategy if subjects are carefully selected, and carefully monitored. Adverse effects will occur only if the usage recommendations based on previous researches are not followed. (Hypertens Res 2006; 29: 211-216)
\end{abstract}

Key Words: aldosterone, angiotensin-converting enzyme inhibitor, angiotensin II type 1 receptor blocker, aldosterone breakthrough, hyperkalemia

\section{Introduction}

The importance of aldosterone as a hormone associated with a risk of cardiovascular events has been the focus of attention recently. Both the Randomized Aldactone Evaluation Study (RALES) (1) and the Eplerenone Post-AMI Heart Failure Efficacy and Survival Study (EPHESUS) (2) found that mortality, risk of hospitalization, and onset of cardiovascular events decreased significantly after added administration of the mineralocorticoid receptor (MR) antagonist spironolactone or eplerenone in patients with severe heart failure, and following acute myocardial infarction complicated with left ventricular dysfunction. These studies have had a major impact in this field, and have contributed to a rapid increase in the volume of MR antagonist prescribed for treating heart failure $(3,4)$. Aldosterone blockade is now also included in certain heart failure treatment guidelines $(5,6)$. These studies

From the ${ }^{1}$ Department of Internal Medicine, International University of Health and Welfare Mita Hospital, Tokyo, Japan; ${ }^{2}$ Department of Internal Medicine, Keio University, Tokyo, Japan; and ${ }^{3}$ Prince Henry's Institute of Medical Research, Clayton, Australia.

Address for Reprints: Atsuhisa Sato, M.D., Ph.D., Department of Internal Medicine, International University of Health and Welfare Mita Hospital, 1-43, Mita, Minato-ku, Tokyo 108-8329, Japan. E-mail: atsu-sa@pb3.so-net.ne.jp

Received August 9, 2005; Accepted in revised form January 13, 2006. 
may have given the impression that only aldosterone blockade was beneficial, but in fact combination therapy with angiotensin-converting enzyme (ACE) inhibitors or angiotensin II (Ang II) type 1 (AT1) receptor blockers (ARB) works best. That is, when using MR antagonist as an "organ protecting drug," further organ protection could be expected by the addition of an ACE inhibitor or ARB. A novel feature of this combination therapy is that it requires only a small dose of MR antagonist and does not induce any reduction in blood pressure. However, warnings have been given recently due to the emergence of adverse effects associated with the combined use of these drugs. The prevailing view since the publication of RALES is that combined use is not recommended because it tends not to be performed correctly (3).

The present review, therefore, attempts to take a fresh look at combination therapy with $\mathrm{MR}$ antagonist and an $\mathrm{ACE}$ inhibitor or ARB from a clinical perspective, and in terms of basic research. If subjects are carefully selected, the combination of MR antagonist and renin-angiotensin (RA) inhibitors should be a useful strategy. Adverse effects are only occurring because the RALES and EPHESUS usage recommendations are not being properly followed.

\section{Background to Combination Therapy}

Important, independent findings concerning MR antagonist combination therapy have been obtained from two different fields. First, predominantly basic research has shown that a minor change in the action of aldosterone mediated by MR in nonepithelial tissues directly causes organ impairment. Secondly, predominantly clinical research has clearly demonstrated an aldosterone breakthrough phenomenon during RA inhibitor therapy. MR antagonists are effective potassiumsparing diuretics that provide a reliable base for treating patients with essential hypertension and primary and secondary aldosteronism, but the concept of aggressively using MR antagonist as an "organ protecting" drug is relatively new. Up to about 10 years ago, aldosterone was only recognized as a classical hormone controlling electrolyte balance in the kidneys. It has since become clear that this is just one of the various actions of aldosterone; for example, aldosterone has been reported to directly impair the heart, blood vessels, brain, kidneys, and other organs, forming vasculitis via nonepithelial $\operatorname{MR}(7)$.

The earliest research on the effects of aldosterone, which was a study on cardiac fibrosis in rats, revealed that aldosterone-induced hypertension and cardiac fibrosis were completely different mineralocorticoid effects. MR antagonist at a dose having little effect on lowering blood pressure could inhibit aldosterone-induced cardiac fibrosis $(8,9)$. This means that even if blood pressure were to be controlled, direct organ damage could be brought on by an inappropriate balance between aldosterone and salt. 11ß-Hydroxysteroid dehydrogenase type 2 (11 $\beta$-HSD2), an enzyme that protects aldosterone MR selectivity, is mostly absent in the heart.
Considering of the concentration of free plasma glucocorticoids, it is believed that glucocorticoids bind to at least $90 \%$ of MR (glucocorticoid-occupied-but-not activated MR), while aldosterone binds to the remaining 10\% (10). However, when the condition becomes pathological by an inappropriate balance between aldosterone and salt, the action of aldosterone is accentuated via MR in the heart (11). Another possibility is that glucocorticoids may play a role in the redox activation of nonepithelial MR (12). When an intracellular redox state is altered by generation of reactive oxygen species, glucocorticoids may become MR agonists. Irrespective of which of these mechanisms is operative, however, only a small dose of an anti-aldosterone drug would be required to protect against the organ damage induced by nonepithelial MR, and such a dose would not be likely to lower blood pressure (13).

One of the major incentives for implementing the RALES trial was the discovery of the aldosterone breakthrough phenomenon. The pilot study for RALES emphasized the importance of aldosterone breakthrough (14). In terms of regulation of aldosterone synthesis, Ang II played a central role, both in AT1 receptor-mediated short-term and long-term regulation. It was thought that, in theory, ACE inhibitors and ARBs could continually inhibit aldosterone production. However, it is becoming apparent that these agents cannot completely inhibit aldosterone production, and it has also become clear that when both drugs are used, regardless of the disease, aldosterone breakthrough generally occurs in some percentage of patients (15-17). The mechanisms of aldosterone breakthrough during the use of RA inhibitors remain obscure (17). The plausible mechanisms are insufficient inhibition of ACE activity, an effect of Ang II independent of ACE activity, and other mechanisms such as potassium, genetics, endothelin-1, and adrenocorticotropic hormone.

To date, clinical studies have shown that ACE inhibitors are useful for protecting organs such as the heart, kidneys, blood vessels, and brain. Evidence that ARBs may also protect organs has recently been increasing. However, the longterm effect of aldosterone was not inhibited in some subjects, so the possibility of organ damage due to breakthrough aldosterone cannot be ignored. Non-epithelial MR-mediated effects played a major role, and they enable inhibition with MR antagonist at a small dose, not lower blood pressure. Therefore, the idea is now to combine a small dose of MR antagonist to an ACE inhibitor or ARB. It must not be forgotten that RALES and EPHESUS both showed that excellent results were obtained by adding an aldosterone blockade agent to a standard therapy centered on ACE inhibitors or ARBs.

\section{RALES and EPHESUS Studies}

The RALES and EPHESUS trials were performed to investigate the "organ protection" conferred by combination therapy with RA inhibitors and MR antagonists. In the Cooperative 
North Scandinavian Enalapril Survival Study (CONSENSUS), which targeted severe heart failure patients, it was reported that prognosis was poor in subjects with a high plasma aldosterone level. Studies of Left Ventricular Dysfunction (SOLVD) also showed in-hospitalization and deaths due to aggravated heart failure in about $48 \%$ of cases despite those cases undergoing ACE inhibitor administration. This meant there was still room for improving treatment for heart failure, so a small-scale study investigating the effects of added administration of MR antagonist was conducted prior to RALES $(18,19)$. However, the 1999 RALES ultimately had the biggest impact in this field (1).

Prior to the present study, a RALES dose-setting study (the pilot study for RALES) was conducted to investigate the optimal dose of spironolactone in subjects who were already taking an ACE inhibitor. The purpose of this was to prevent hyperkalemia, which is of the utmost concern in combination therapy with ACE inhibitors and MR antagonists (14). Urinary aldosterone was shown to increase, and atrial natriuretic factor (ANF) was shown to significantly decrease from the observation period dose-dependently after spironolactone administration, which meant that neural and humoral factors in heart failure should improve. Also, it was indicated that if the dose were set to 12.5 to $25 \mathrm{mg} /$ day (recommended doses), serious hyperkalemia would be avoided. Instructions were also given during the study to periodically check the serum potassium level. The spironolactone dose was to be reduced in the event hyperkalemia was noted $(25 \mathrm{mg}$ administered every other day). Thereafter, the importance of this strict potassium check, and the regulation of the spironolactone dose, were clearly ignored in most clinical settings, and for this reason serious adverse effects have been consistently reported (3).

Thus, RALES first raised the importance of aldosterone breakthrough, and then looked at a strategy by which MR antagonist could be added to completely block the effect of the aldosterone. However, the dose was set at $25 \mathrm{mg}$ due to the risk of hyperkalemia, and this is a considerably smaller dose compared to the dose of spironolactone normally employed. It was also not clear whether there would be a diuretic effect (regardless of the excreted urinary sodium volume), so the dose was one that would not have an effect on hemodynamics (14). However, the fact that mortality and hospitalization rates significantly decreased at this dose were clinical proof that MR blockade can be very beneficial, even at a dose that does not lower blood pressure. What was obtained from RALES was the precondition that standard treatment was already able to be conducted for heart failure centered on ACE inhibitors, and that aldosterone blockade was indicated for the first time, and was extremely useful in such an event.

In the EPHESUS trial as well, the reduction in the overall mortality rate due to eplerenone was best when an ACE inhibitor or ARB and $\beta$-blocker were used in combination (2). That is, EPHESUS showed for the first time the beneficial effects of an MR antagonist when it is administered to acute myocardial infarction patients with left ventricular dysfunction who are already on appropriate standard therapy. As with RALES, caution had to be exercised to avoid hyperkalemia, so patients with a serum potassium level of at least $5.0 \mathrm{mEq} / 1$ and a serum creatinine level of at least $2.5 \mathrm{mg} / \mathrm{dl}$ were excluded. In RALES, only $10 \%$ of subjects used a $\beta$-blocker, but in EPHESUS $\beta$-blockers were prescribed to $75 \%$ of subjects. In that sense the EPHESUS study showed how it was possible to use $\beta$-blockers and MR antagonists safely in combination without any serious adverse effects. Incidentally, in a study in which a $\beta$-blocker (carvedilol or metoprolol) was also administered to heart failure patients being treated with an ACE inhibitor, no significant decrease in the plasma aldosterone concentration was seen when the patients were followed for 52 weeks (20). It is therefore thought that combination therapy with MR antagonist is indeed required even during $\beta$-blocker administration in order to reliably inhibit the action of aldosterone.

There have been no concrete studies concerning how long aldosterone blockade should be continued. However, Pitt et $a l$. have suggested that it should be acceptable to stop administration once the ejection fraction (EF) reaches $40 \%$, and disease signs disappear (21). The same study showed that about one-third of cases were complicated with diabetes mellitus (DM), so further sub-analysis on only these cases would be of use. We previously reported that the effects of added administration of spironolactone were better in chronic renal disease patients whose underlying disease was DM (22). A 4E-left ventricular hypertrophy study found that when eplerenone, enalapril, and the two in combination were administered to essential hypertension patients with left ventricular hypertrophy, the combination group showed the greatest reduction in cardiac hypertrophy (21).

The mechanism of aldosterone breakthrough is still not known (17), but sub-analysis of the Valsartan Heart Failure Trial (Val-Heft) revealed that the plasma aldosterone concentration did not differ significantly whether an ACE inhibitor was administered or not (23). This suggests that even when an ACE inhibitor is administered in combination with an ARB, aldosterone is not inhibited further. Aldosterone breakthrough was also seen using a combination of ACE inhibitor and ARB in the Randomized Evaluation of Strategies for Left Ventricular Dysfunction (RESOLVD) Pilot Study (24). There was no detailed investigation of dose, but in both studies the RA inhibitor dose was certainly not low. With current RA inhibitors, such as when both are used in combination, aldosterone cannot be reliably inhibited in some cases. A review by Azizi and Menard also surmised that further reduction in the plasma aldosterone concentration would be unlikely even if an ACE inhibitor and ARB were administered in combination (25). It is possible that the added administration of a small dose of MR antagonist in such cases would be effective. 


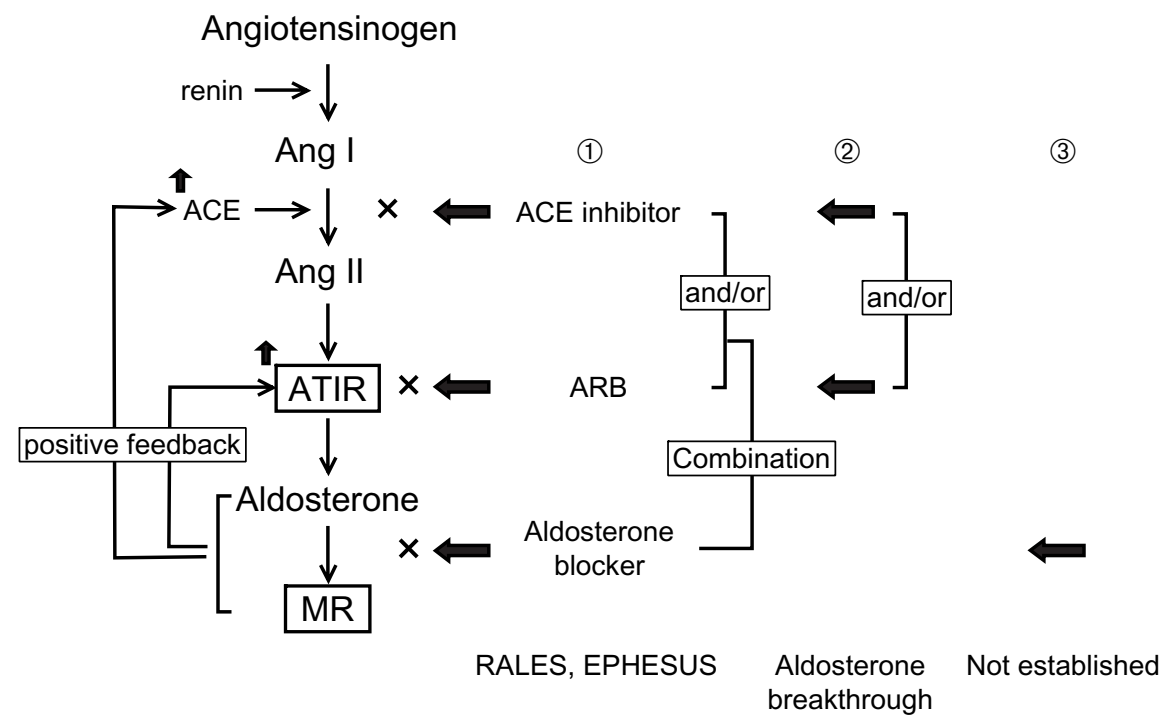

Fig. 1. The renin-angiotensin-aldosterone (RAA) system and aldosterone blockade and renin-angiotensin inhibitors (ACE inhibitors and ARBs). The RAA has positive feedback systems, and thus it is more effective to block it at several points. It is very important to note that at present we do not have any clinical evidence of the effects of aldosterone blockade alone in patients with chronic heart failure. Ang I, angiotensin I; Ang II, angiotensin II; AT1R, angiotensin II type 1 receptors; MR, mineralocorticoid receptors; ACE, angiotensin-converting enzyme; ARB, angiotensin II type 1 receptor blocker; RALES, the Randomized Aldactone Evaluation Study; EPHESUS, the Eplerenone Post-AMI Heart Failure Efficacy and Survival Study.

\section{Hyperkalemia in Combination Therapy}

Concomitant administration of an RA inhibitor and a small dose of MR antagonist could be a reasonably good strategy for protecting the organs, but there has been a recent succession of reports in which hyperkalemia was caused by such combination therapies. However, other studies including RALES and EPHESUS have demonstrated that adverse effects should not be a problem if subjects are carefully chosen. Although hyperkalemia is again the focus here, it is extremely important that the serum potassium level does not fall, especially when treating heart failure. In the Systolic Hypertension in the Elderly Program (SHEP) study, which targeted elderly hypertensive patients, it was reported that although the blood pressure lowering effect through the diuretic chlortalidone was confirmed when hypokalemia occurred, the effect of preventing cardiovascular events disappeared. Here we will introduce some reports on hyperkalemia, and examine the proper way of administering both agents in combination.

After RALES was published, Juurlink et al. reported that the use of spironolactone in heart failure patients rapidly increased, but that in-hospitalization due to hyperkalemia and deaths associated with hyperkalemia also similarly increased (3). They also warned that added administration of spironolactone would not significantly improve re-hospitalization or overall mortality rates among heart failure patients. They attributed this to the following causes: a lack of careful mon- itoring of potassium levels during the course of the disease (as distinct from the very careful potassium monitoring in RALES and EPHESUS), a failure to review renal dysfunction carefully enough, too high a dose of spironolactone, a failure to stop administering potassium to prevent hypokalemia caused by diuretics, and a failure to instruct patients to avoid a large amount of potassium in their diets.

In an analysis by Bozkurt et al., most subjects who were administered spironolactone after RALES did not have severe heart failure, but rather one-third of them had renal failure, and at least one-third were prescribed potassium at the same time (26). Based on their report, it is evident that a large discrepancy exists between the clinical trial and actual practice. That is, the use of spironolactone in actual practice differs largely from the way the drug was used in the clinical trials. Svensson et al. reported that the administration of spironolactone in congestive heart failure patients caused more adverse effects than had been expected (27). Hyperkalemia and uremia were reportedly prevalent especially in the elderly and patients with lower left ventricular EF and higher NYHA function classes. Eplerenone is associated with few endocrine adverse effects, but the incidence of hyperkalemia is dosedependent, so its administration, like the administration of spironolactone, should be monitored. Wrenger et al. analyzed 44 cases of hyperkalemia at their institution, and reported problems in the elderly and with spironolactone at high doses (28). They also reported that $80 \%$ of cases were complicated with type 2 DM. Schepkens et al. analyzed 25 patients with hyperkalemia (at least $6 \mathrm{mEq} / \mathrm{l}$ ) who were urgently hospital- 
ized, and reported that advanced age, renal failure, complication with type $2 \mathrm{DM}$, worsening heart failure, dehydration, and concomitant therapy with another agent that would cause hyperkalemia were all factors for which caution should be exercised (29). Tamirisa et al. found that added administration of spironolactone in heart failure patients caused more adverse effects of hyperkalemia and renal dysfunction than had previously been reported (29). They also reported that being elderly, having type $2 \mathrm{DM}$, and having a high potassium level at the start of administration were factors that contributed to hyperkalemia (30). It has been pointed out that subjects presenting with hyperkalemia are often also on $\beta$ blocker medication, so it is surmised that $\beta$-blockers aggravate hyperkalemia caused by spironolactone. However, the results of concomitant administration with $\beta$-blockers differ considerably from those of EPHESUS.

Taken together, these reports demonstrate the importance of fulfilling the eligibility criteria of RALES and EPHESUS when providing concomitant therapy with spironolactone and an RA inhibitor. In RALES, the incidence of hyperkalemia did not actually differ from that in the placebo group. That is because if subjects are selected and followed closely, the potassium level will rarely become problematic, and because the high incidence of adverse effects seen was due to incorrect usage. Also, elderly patients or those with dehydration, renal dysfunction, and aggravated heart failure require further close monitoring or termination of spironolactone administration. It is also important to discontinue drugs that elevate the potassium level, and closely monitor the intake of dietary potassium. Sligl et al. reported that if these factors were taken care of and a specialist provided follow up, then there would be merit to spironolactone administration even in patients who did not fulfill the RALES eligibility criteria (4). There has still been no consensus on whether or not complication with type 2 DM contributes to the onset of hyperkalemia, so further investigation is required on that point.

We previously reported that adding $25 \mathrm{mg}$ of spironolactone to the ACE inhibitor trandolapril in patients with proteinuria of at least $0.5 \mathrm{~g}$ /day irrespective of the success of blood pressure control had the effect of decreasing proteinuria independently of blood pressure (21). In that study, none of the subjects discontinued spironolactone due to hyperkalemia. That was probably because no more than $25 \mathrm{mg}$ of spironolactone was used according to the result of urine testing, which confirmed that the mean glomerular filtration rate was $93 \pm 5 \mathrm{ml} / \mathrm{min}$ (the minimal rate of $85 \mathrm{ml} / \mathrm{min}$ ) in all subjects whose ages ranged from 52 to 63 years. These factors are all consistent with the previously reported precautions to prevent hyperkalemia.

\section{Conclusion}

Given the clinical relevance of aldosterone breakthrough, adding MR antagonist to RA inhibitors should be a useful strategy in terms of organ protection. It must not be forgotten that RALES and EPHESUS both showed that excellent results were obtained by adding an aldosterone blockade agent to a standard therapy centered on ACE inhibitors or ARBs, and that we do not have any evidence at present that an MR antagonist alone is effective for the treatment of chronic heart failure. We summarize these results in Fig. 1. To attain favorable clinical results, it is important that the eligibility criteria of RALES and EPHESUS be fulfilled when using the combination therapy. If subjects are selected and followed closely, the adverse effects, especially hyperkalemia, will rarely become problematic.

\section{References}

1. Pitt B, Zannad F, Remme WJ, et al, for the Randomized Aldactone Evaluation Study: The effect of spironolactone on morbidity and mortality in patients with severe heart failure. N Engl J Med 1999; 341: 709-717.

2. Pitt B, Remme W, Zannad F, et al, for the Eplerenone PostAcute Myocardial Infarction Heart Failure Efficacy and Survival Study Investigators: Eplerenone, a selective aldosterone blocker, in patients with left ventricular dysfunction after myocardial infarction. $N$ Engl J Med 2003; 348: 1309 1321.

3. Juurlink DN, Mamdani MM, Lee DS, et al: Rates of hyperkalemia after publication of the Randomized Aldactone Evaluation Study. N Engl J Med 2004; 351: 543-551.

4. Sligl W, McAlister FA, Ezekowitz J, Armstrong PW: Usefulness of spironolactone in a specialized heart failure clinic. Am J Cardiol 2004; 94: 443-447.

5. Greenberg B: Treatment of heart failure: state of the art and prospectives. J Cardiovasc Pharmacol 2002; 38: 59-63.

6. Remme WJ, Swedberg K: Comprehensive guideline for the diagnosis and treatment of chronic heart failure. Eur $J$ Heart Fail 2002; 4: 11-22.

7. Rocha R, Funder JW: The pathophysiology of aldosterone in the cardiovascular system. Ann N Y Acad Sci 2002; 970: 89-100.

8. Young M, Head G, Funder JW: Determinants of cardiac fibrosis in experimental hypermineralocorticoid states. $\mathrm{Am} \mathrm{J}$ Physiol 1995; 269: 657-662.

9. Brilla CG, Weber KT: Mineralocorticoid excess, dietary sodium and myocardial fibrosis. J Lab Clin Med 1992; 120: 893-901.

10. Funder JW: Aldosterone, salt and cardiac fibrosis. Clin Exp Hypertens 1997; 19: 885-899.

11. Sato A, Saruta T: Aldosterone-induced organ damage: plasma aldosterone level and inappropriate salt status. Hypertens Res 2004; 27: 303-310.

12. Funder JW: Is aldosterone bad for the heart? Trends Endocrinol Metab 2004; 15: 139-142.

13. Sato A, Suzuki Y, Saruta T: Effects of spironolactone and angiotensin-converting enzyme inhibitor on left ventricular hypertrophy in patients with essential hypertension. Hypertens Res 1999; 22: 17-27.

14. The RALES Investigators: Effectiveness of spironolactone added to an angiotensin-converting enzyme inhibitor and a loop diuretic for severe chronic congestive heart failure (the Randomized Aldactone Evaluation Study). Am J Cardiol 
1996; 78: 902-907.

15. Sato A, Saruta T: Aldosterone escape during angiotensinconverting enzyme inhibitor therapy in essential hypertensive patients with left ventricular hypertrophy. $J$ Int Med Res 2001; 29: 13-21.

16. Sato A, Hayashi K, Naruse M, Saruta T: Effectiveness of aldosterone blockade in patients with diabetic nephropathy. Hypertension 2003; 41: 64-68.

17. Sato A, Saruta T: Aldosterone breakthrough during angiotensin-converting enzyme inhibitor therapy. Am J Hypertens 2003; 16: 781-788.

18. van Vliet AA, Donker AJM, Nauta JJP, Verheugt FWA: Spironolactone in congestive heart failure refractory to high-dose loop diuretic and low-dose angiotensin-converting enzyme inhibitor. Am J Cardiol 1993; 71: 21A-28A.

19. Barr CS, Lang CC, Hanson J, Arnott M, Kennedy N, Struthers AD: Effects of adding spironolactone to an angiotensinconverting enzyme inhibitor in chronic congestive heart failure secondary to coronary artery disease. Am J Cardiol 1995; 76: 1259-1265.

20. Fung JWH, Yu CM, Yip G, et al: Effect of beta blockade (carvedilol or metoprolol) on activation of the renin-angiotensin-aldosterone system and natriuretic peptides in chronic heart failure. Am J Cardiol 2003; 92: 406-410.

21. Pitt B, Reichek N, Willenbrock R, et al: Effects of eplerenone, enalapril, and eplerenone/enalapril in patients with essential hypertension and left ventricular hypertrophy: the 4E-left ventricular hypertrophy study. Circulation 2003; 108: 1831-1838.

22. Sato A, Hayashi K, Saruta T: Antiproteinuric effects of mineralocorticoid receptor blockade in patients with chronic renal disease. Am J Hypertens 2005; 18: 44-49.

23. Cohn JN, Anand IS, Latini R, et al, for Valsartan Heart Failure Trial Investigators: Sustained reduction of aldosterone in response to the angiotensin receptor blocker valsartan in patients with chronic heart failure: results from the Valsar$\tan$ Heart Failure Trial. Circulation 2003; 108: 1306-1309.

24. McKlelvie RS, Yusuf S, Mmath P, et al, The RESOLVD Pilot Study Investigators: Comparison of candesartan, enalapril, and their combination in congestive heart failure. Randomized Evaluation of Strategies for Left Ventricular Dysfunction (RESOLVD) pilot study. Circulation 1999; 100: $1056-1064$.

25. Azizi M, Menard J: Combined blockade of the renin-angiotensin system with angiotensin-converting enzyme inhibitors and angiotensin II type 1 receptor antagonists. Circulation 2004; 109: 2492-2499.

26. Bozkurt B, Agoston I, Knowlton AA: Complications of inappropriate use of spironolactone in heart failure: when an old medicine spirals out of new guidelines. J Am Coll Cardiol 2003; 41: 211-214.

27. Svensson M, Gustafsson F, Galatius S, Hildebrandt PR, Atar D: Hyperkalemia and impaired renal function in patients taking spironolactone for congestive heart failure: retrospective study. BMJ 2003; 327: 1141-1142.

28. Wrenger E, Muller R, Moesenthin M, Welte T, Frolich JC, Neumann KH: Interaction of spironolactone with ACE inhibitors or angiotensin receptor blockers: analysis of 44 cases. BMJ 2003; 327: 147-149.

29. Schepkens H, Vanholder R, Billiouw JM, Lameire N: Lifethreatening hyperkalemia during combined therapy with angiotensin-converting enzyme inhibitors and spironolactone: an analysis of 25 cases. Am J Med 2001; 110: 438441.

30. Tamirisa KP, Aaronson KD, Koelling TM: Spironolactoneinduced renal insufficiency and hyperkalemia in patients with heart failure. Am Heart J 2004; 148: 971-978. 